\title{
OPEN Prospective, comparative clinical pilot study of cold atmospheric plasma device in the treatment of atopic dermatitis
}

\author{
Young Jae Kim, Dong Jun Lim, MiYoung Lee, Woo Jin Lee, Sung Eun Chang \& \\ Chong Hyun Won $\bowtie$
}

Cold atmospheric plasma generates free radicals through the ionization of air at room temperature. Its effect and safety profile as a treatment modality for atopic dermatitis lesions have not been evaluated prospectively enough. We aimed to investigate the effect and safety of cold atmospheric plasma in patients with atopic dermatitis with a prospective pilot study. Cold atmospheric plasma treatment or sham control treatment were applied respectively in randomly assigned and symmetric skin lesions. Three treatment sessions were performed at weeks 0,1 , and 2 . Clinical severity indices were assessed at weeks $0,1,2$, and 4 after treatment. Additionally, the microbial characteristics of the lesions before and after treatments were analyzed. We included 22 patients with mild to moderate atopic dermatitis presented with symmetric lesions. We found that cold atmospheric plasma can alleviate the clinical severity of atopic dermatitis. Modified atopic dermatitis antecubital severity and eczema area and severity index score were significantly decreased in the treated group. Furthermore, scoring of atopic dermatitis score and pruritic visual analog scales significantly improved. Microbiome analysis revealed significantly reduced proportion of Staphylococcus aureus in the treated group. Cold atmospheric plasma can significantly improve mild and moderate atopic dermatitis without safety issues.

Artificial plasma has been used in the tissue removal, cauterization, or sterilization of medical instruments, being a technology with potential to be applied in various medical fields ${ }^{1}$. In particular, non-thermal atmospheric plasma, also known as cold atmospheric plasma (CAP) was getting attention due to its possibility to directly contact with the human body. In this context, CAP allows heat-sensitive targeting applications at low temperatures, without any specific extra cooling. As above-mentioned, CAP serves different medical purposes as a disinfecting and bleaching through the generation of free radicals including the reactive oxygen species or nitrogen oxygen species in combination with transient electric fields or UV radiation ${ }^{2-4}$. Recent studies updated that CAP promotes the wound healing process by reducing inflammation and oxidative stress ${ }^{5,6}$. Its potential roles in promoting angiogenesis, inducing proliferation, tissue oxygenation and migration of keratinocytes and fibroblasts have been also documented ${ }^{7-9}$. Moreover, CAP is also effective for relieving skin inflammation in mice with atopic dermatitis ${ }^{10,11}$.

Atopic dermatitis is a common chronic inflammatory skin disease that affects $10-20 \%$ of children and $1-3 \%$ of adults ${ }^{12}$. Since the severity of each individual varies, a systematic, and diverse therapeutic approach is required according to the patients' clinical manifestations. Nonetheless, its known that several therapies, such as topical agents or oral medications still have potential side effects, especially if they are used for a long term. In addition, even under an appropriate treatment, most atopic lesions are likely to worsen rather than completely disappear like an external flame that never goes out. Therefore, an alternatively sustainable treatment without patients' burden is urged to manage the localized atopic lesions. In this context, CAP device may serve as a novel treatment option for these indications.

Until now, limited studies are available regarding the clinical evidence of CAP treatment in atopic dermatitis patients ${ }^{13,14}$. This pilot study investigated the efficacy and safety of CAP in atopic dermatitis patients. 
a

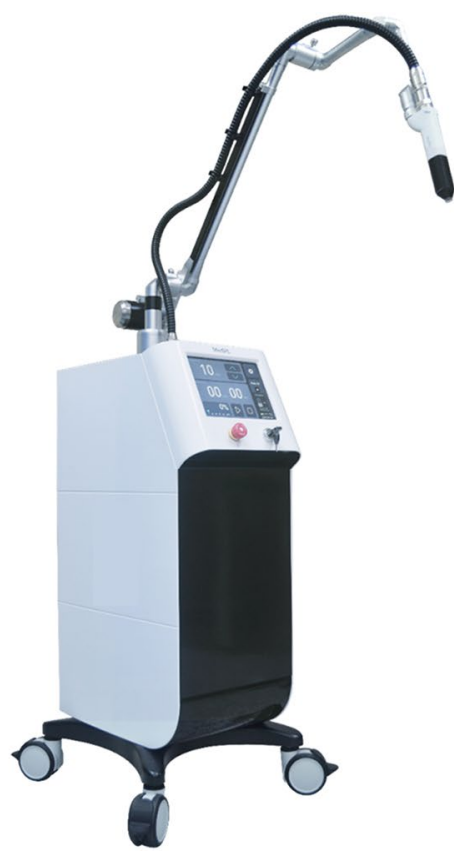

b

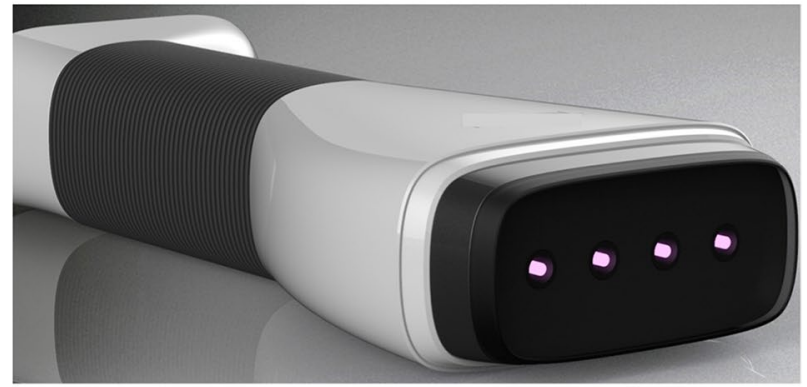

Figure 1. Cold atmospheric plasma (CAP) treatment device in this study, which uses argon gas to generate plasma. (a) Overall device appearance (b) A four-jet array handpiece was used in this study.

\section{Methods}

Patients. This study was approved by the institutional review board of Asan Medical Center (2020-0811). A prospective clinical study was performed, from May 20, 2020, to August 10, 2020. All the patients signed the informed patient. This study included patients with an age above 19 and with a diagnosis of atopic dermatitis of mild to moderate severity with symmetric skin lesions. The severity of disease was defined as "mild" if their Investigator's Global Assessment (IGA) score 2 and "moderate" if their IGA score 3 (Supplementary Table 1). Moreover, only patients who had atopic dermatitis lesions with total body surface area (BSA) $<30 \%$ were included. The exclusion criteria included any history of systemic treatment of atopic dermatitis, such as corticosteroids, antibiotics, immunosuppressive agents, or phototherapy used within the last 4 weeks prior to the study. Any history of topical agents, such as topical corticosteroids, immunosuppressants, or antibiotics within 2 weeks also excluded. Patients who had other severe medical problems or mental illness, and who were pregnant were excluded from this study. Patients were only allowed to take oral antihistamines $(n=3)$ for the relief of symptom and to apply emollients during the whole study period.

Plasma device and treatment protocol. The device used during the study was a microwave-driven CAP device called MediPL Derm, developed by MediPL (Seoul, Korea) (Fig. 1a). This CAP device belongs to the indirect plasma type entrained with an argon gas flow ${ }^{15,16}$. A sham treatment device was also prepared, having the same appearance of the genuine plasma. CAP device employed the following setting to operate: microwave frequency $2.45 \mathrm{GHz} \pm 50 \mathrm{MHz}$, plasma power with $1.5 \mathrm{~W}$ at each electrode array, plasma temperature between the plasma device and target $<41{ }^{\circ} \mathrm{C}$ to prevent low temperature burn, continuous signal, four-array jet handpiece (Fig. 1b). Since the plasma intensity can vary depending on the flow rate, we maintained argon gas flow as 0.6 standard liter per minute $(\mathrm{slm})$ per electrode to maintain a constant plasma generation. The change in the total ultraviolet index before and after using the plasma device was 0.0055 . Supplementary Fig. 1 shows optical emission spectrum of plasma device in this study. Sham treatment device employed the same setting as the CAP device, except for the absence of microwave for ignition and for that reason no plasma energy generates.

For each patient, CAP or sham treatment was randomly assigned and used to treat the symmetric lesions. We defined symmetric lesions as the skin lesions existing on both sides of anatomical sites of extremities. For instance, if the patients with atopic dermatitis $(\mathrm{AD})$ lesions on their both arms are randomly assigned to receive plasma treatment on the right arm, the patients will receive sham treatment on the left arm. Before the treatment, we wiped off the lesions with a normal saline soaked gauze. The plasma or sham treatment device was set to keep the distance between the lesion and device within $5 \mathrm{~mm}$. The treatments were made during five minutes in each session.

Plasma and sham treatment were weekly used at week 0 (baseline), 1 and 2, being asked the patients to visit at week 4 for follow-up assessment.

Assessment of clinical severity in atopic dermatitis. At week $0,1,2$, and 4 , all the patients of IGA were assessed with a modified ADAS (Atopic Dermatitis Antecubital Severity) score, EASI (Eczema Area and Severity Index) score, SCORAD (Scoring of AD) score, and pruritic visual analog scale (VAS) score. The modified ADAS (Atopic Dermatitis Antecubital Severity) score is calculated by multiplying the intensity of inflam- 


\begin{tabular}{|l|l|l|l|l|l|l|}
\hline \multirow{2}{*}{ Patient no. } & \multirow{2}{*}{ Sex/age } & \multicolumn{3}{l}{ Baseline } & \multicolumn{2}{l}{} \\
\cline { 3 - 6 } & IGA (Lt/Rt) & Severity & EASI & Total serum IgE (kU/I) & Treatment site \\
\hline 1 & $\mathrm{~F} / 29$ & $3 / 3$ & Moderate & 5.2 & 246 & Upper arm \\
\hline 2 & $\mathrm{M} / 23$ & $3 / 3$ & Moderate & 5.6 & 3786 & Popliteal area \\
\hline 3 & $\mathrm{M} / 41$ & $3 / 3$ & Moderate & 2.7 & 72.8 & Shin \\
\hline 4 & $\mathrm{M} / 23$ & $3 / 3$ & Moderate & 7.5 & 1454 & Antecubital area \\
\hline 5 & $\mathrm{~F} / 39$ & $2 / 2$ & Mild & 1.6 & 25 & Medial knee \\
\hline 6 & $\mathrm{~F} / 22$ & $3 / 3$ & Moderate & 2.4 & 671 & Antecubital area \\
\hline 7 & $\mathrm{~F} / 23$ & $3 / 3$ & Moderate & 8.8 & 124 & Popliteal and antecubital area \\
\hline 8 & $\mathrm{~F} / 19$ & $2 / 2$ & Mild & 2.6 & 116 & Popliteal and antecubital area \\
\hline 9 & $\mathrm{M} / 33$ & $2 / 2$ & Mild & 1.0 & 2684 & Upper arm \\
\hline 10 & $\mathrm{~F} / 22$ & $3 / 3$ & Moderate & 3.4 & $<2$ & Antecubital area \\
\hline 11 & $\mathrm{~F} / 25$ & $2 / 2$ & Mild & 0.8 & 54.3 & Antecubital area \\
\hline 12 & $\mathrm{~F} / 32$ & $3 / 3$ & Moderate & 7.6 & 3901 & Antecubital area \\
\hline 13 & $\mathrm{~F} / 22$ & $3 / 3$ & Moderate & 2.4 & 662 & Antecubital area \\
\hline 14 & $\mathrm{M} / 20$ & $3 / 3$ & Moderate & 9.4 & 2714 & Antecubital area \\
\hline 15 & $\mathrm{M} / 24$ & $2 / 3$ & Mild/moderate & 4.0 & 645 & Antecubital area \\
\hline 16 & $\mathrm{M} / 28$ & $3 / 3$ & Moderate & 5.3 & 2241 & Antecubital area \\
\hline 17 & $\mathrm{M} / 32$ & $3 / 3$ & Moderate & 10 & 1496 & Antecubital area and elbow \\
\hline 18 & $\mathrm{~F} / 42$ & $2 / 2$ & Mild & 4.6 & $>5000$ & Popliteal and antecubital area \\
\hline 19 & $\mathrm{M} / 31$ & $2 / 2$ & Mild & 2.4 & 2737 & Antecubital area \\
\hline 20 & $\mathrm{~F} / 28$ & $2 / 2$ & Mild & 1.4 & 1116 & Antecubital area \\
\hline 21 & $\mathrm{~F} / 20$ & $2 / 2$ & Mild & 2.4 & 63.4 & Shin \\
\hline 22 & $\mathrm{~F} / 21$ & $2 / 2$ & Mild & 2.2 & 37.2 & Popliteal area \\
\hline & & & & & & \\
\hline
\end{tabular}

Table 1. Characteristics of the patients.

mation by the area of an antecubital eczematous lesion ${ }^{17}$. The intensity of inflammation is evaluated using a 6 -grade scale ( 0 , absence; 1 , mild; 2 , mild-moderate; 3 , moderate; 4 , severe; and 5 , very severe) for erythema, scale and excoriation on skin lesion respectively. The visual analog scale (VAS) is a scale consisting of $100 \mathrm{~mm}$ long line and a single question. In pruritus VAS, we asked every patient the severity of subjective pruritus in last three days. Scale 0 means "never felt itching sense", otherwise scale 10 means "most severe itching sense". Then the patient was asked to mark on the scale as they experienced ${ }^{18}$. The improvement achieved was compared (the degree of improvement) between the treatment group and the control group after treatments. Transepidermal water loss (TEWL) was assessed in patients with AD lesions using tewameter and corneometer (CourageKhazaka, Köln, Germany) before the treatment (week 0) and at week 4 . The clinical effectiveness of CAP was evaluated by comparison with the CAP treated arm and the sham treated counterpart using IGA, modified ADAS score, and TEWL. In addition, overall clinical severity of each patient was also assessed before and after the treatment, using EASI score, SCORAD score, and VAS score.

Skin microbiome study. We swiped the lesions with an aseptic cotton swab for a minute to acquire the sample for microbial analysis. We collected patients' bacterial samples just before the first treatment session (week 0) and at week 4. Microbial analysis was done through 16s-rRNA gene sequencing.

Statistics. Data were analyzed using SPSS 21.0. Paired t-test was used for continuous variables analysis, such as modified ADAS score, EASI score, SCORAD score, pruritus VAS score and Staphylococcus aureus (S. aureus) loading. Chi-square test was used for the analysis of categorical variables, namely proportion of IGA improvement.

\section{Result}

Twenty-two (13 females and 9 males) Korean patients were enrolled in the present study. The patients included in this study completed three treatment sessions and follow-up visit. The mean patient age was $27.2 \pm 6.9$ years (range, 20-42 years). Among 22 patients, 9 patients revealed a mild AD and 13 patients were moderate AD (Table 1). The mean EASI score of mild AD patients was $2.11 \pm 1.14$. The mean EASI score of moderate AD patients was $5.72 \pm 2.71$.

Several clinical indexes showed a statistically significant improvement after CAP treatment compared to sham treatment (Figs. 2, 3). The mean modified ADAS (mean \pm standard deviation) score at baseline was $33.73 \pm 21.21$ in CAP group and $35.02 \pm 15.13$ in sham group, respectively. Along with the study, the mean modified ADAS score in CAP group was decreased to $21.56 \pm 16.07$ at week 1 and $17.23 \pm 18.54$ at week 2 . After three treatment sessions, at week 4 , the mean modified ADAS score in CAP group was 13.12 \pm 15.92 . CAP treated atopic skin lesions showed a significant improvement in modified ADAS score at week 4 ( $p$ value $<0.001$ ) compared to sham 

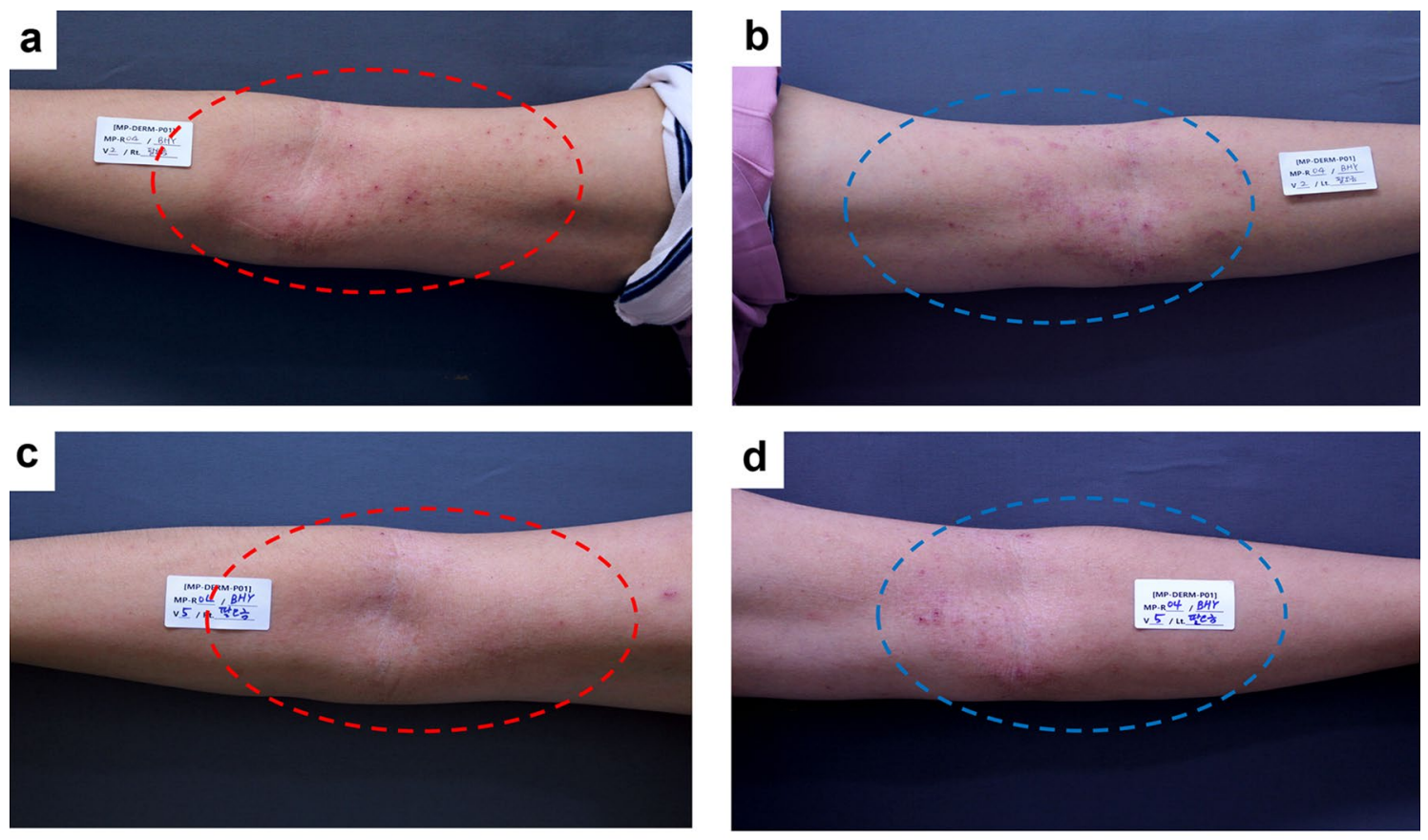

Plasma

Sham

Figure 2. Three treatment sessions of cold atmospheric plasma improved the severity of atopic skin lesion. A 23-years old male with favorable results after CAP treatment compared to sham treated arm. Erythema and papules/indurations noticeably diminished. (a) plasma treated arm, at week 0 (b) sham treated arm, at week 0 (c) plasma treated arm, at week 4 (d) sham treated arm, at week 4.

treated lesion, which showed no statistically significant improvement ( $p$ value $=0.114$ ) (Table 2$)$. The mean EASI score at baseline was $4.24 \pm 2.82$. This value gradually decreased to $3.70 \pm 2.23$ at week 1 and $3.05 \pm 2.22$ at week 2. At the end of the study, the mean EASI score was $2.76 \pm 2.18$, being significantly improved as compared to baseline ( $p$ value $=0.002$ ) (Fig. 4 ). Another atopic severity score, SCORAD had also been significantly improved. The mean SCORAD score at baseline was $29.63 \pm 9.42$, which significantly decreased as much as $19.43 \pm 11.50$ at the end of study ( $p$ value $=0.001$ ). Concerning the pruritic VAS, it was significantly improved at the end of study from $5.14 \pm 1.97$ at baseline to $3.83 \pm 2.12$ ( $p$ value $=0.032)$.

The proportion of lesions that showed an improved IGA score at week 4 was $68.2 \%(15 / 22)$ in CAP group and $40.9 \%(9 / 22)$ in sham group. Although without a statistically significant difference, there was a tendency to improve of IGA score after CAP treatments $(p$ value $=0.069)$. The mean TEWL at plasma treated arms values were not significantly changed, from the baseline $24.40 \pm 11.93$ to $24.24 \pm 7.51 \mathrm{~g} \mathrm{~h}^{-1} \mathrm{~m}^{-2}$ at week 4 ( $p$ value $\left.=0.960\right)$. The decrease in the mean value of corneometer was not statistically significant as well, from $45.71 \pm 13.47$ at baseline to $40.57 \pm 8.94$ at 4 weeks ( $p$ value $=0.133$ ). Notably, when the groups were divided according to total serum IgE levels, the group with total serum $\operatorname{IgE}>100 \mathrm{kU} / \mathrm{I}$ showed a significant decrease in modified ADAS score $(p$ value $<0.001)$ and EASI score $(p$ value $=0.002)$ after the treatment of CAP at final follow-up assessment. However, the group with a total $\mathrm{IgE} \leq 100 \mathrm{kU} / \mathrm{I}$ revealed no significant improvement in the EASI score at final follow-up assessment $(p$ value $=0.730)$.

Concerning the microbial analysis, only nine patients completed the study due to the specimen adequacy and due to the refusal of some patients. We focused on the effect of CAP on the proportion of $S$. aureus over the whole microbiata on the lesion. The mean proportion of S. aureus at baseline was $23.03 \%$ in CAP group, being $16.26 \%$ in sham group. After the treatment sessions, the mean proportion of $S$. aureus was $10.14 \%$ in CAP group, while $15.29 \%$ in sham group. The difference in the reduction of $S$. aureus counting was statistically significant between CAP and sham group ( $p$ value $=0.047$ ).

Side effects were monitored by the investigators (YJK, CHW) during every visit through the physical examination. During the treatment and the follow-up period, no severe side effects were observed, including burn, prolonged erythema, and pain related to CAP treatment. None expressed slight warmth on the treated lesion after the CAP treatments was reported.

\section{Discussion}

$\mathrm{AD}$ occurs as a result of complex interactions, such as skin barrier dysfunction, immunologic dysfunction, environmental, and genetic factors ${ }^{19}$. The chronic course of AD manifested with dry and itchy skin presentation may severely deteriorate the patients' quality of life. In particular, vicious cycles of the scratch and itch cycle cause a continuous mechanical damage to the skin, accelerating several inflammatory reactions, which contributes to a prolonged course of the disease $\mathrm{e}^{20}$. Therefore, stopping the repetitive scratch and itch cycles and restoring the damaged skin barrier may be an effective therapeutic strategy for itchy atopic skin. Notably, it has 


\section{CAP treatment}
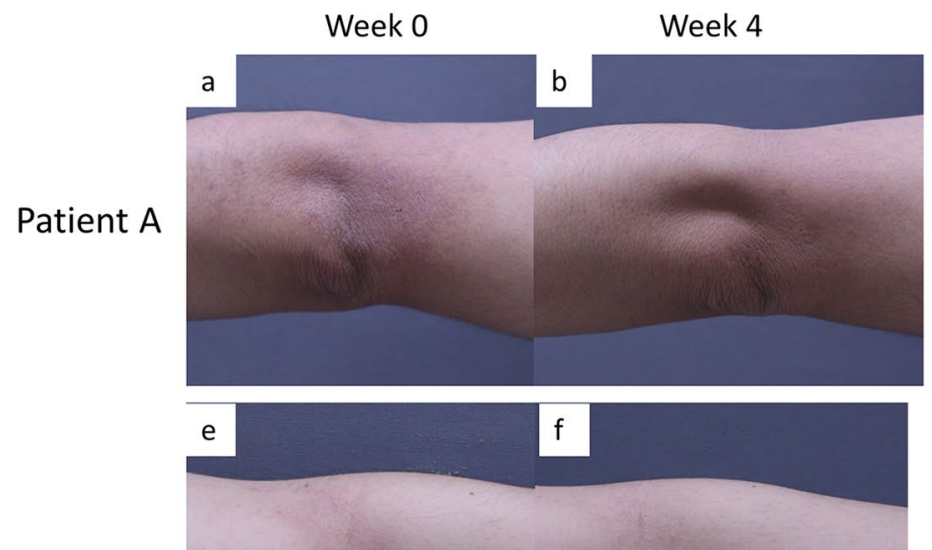

Patient B
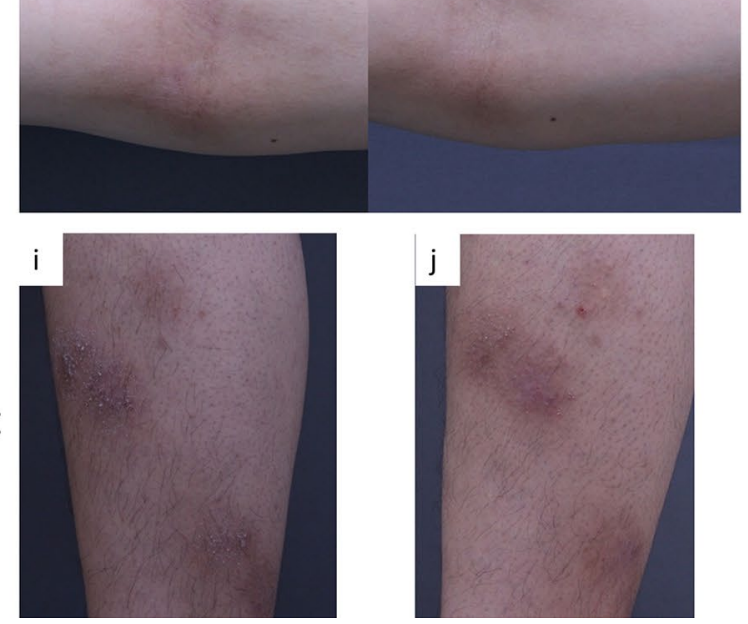

\section{Sham treatment}
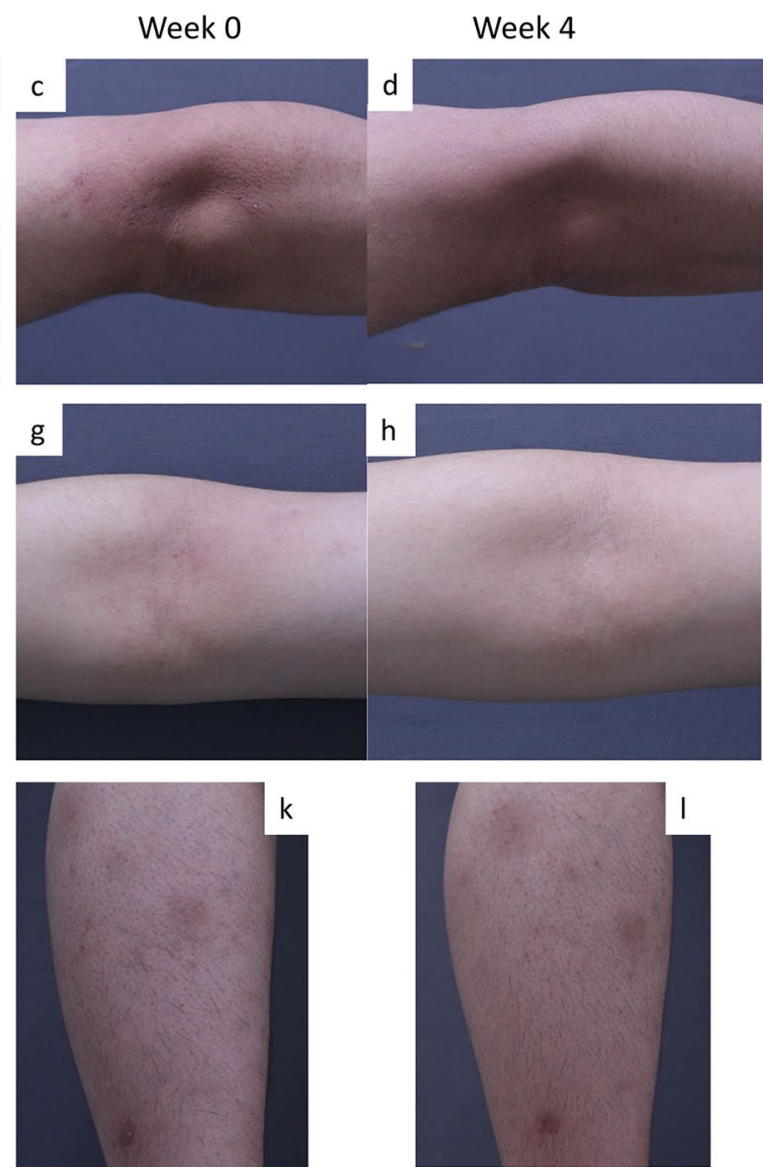

Figure 3. Clinical photography of representative subjects. (a-d) Patient A, 32-years old man with modified ADAS score 85 on his CAP treated arm at week 0 . After three treatment sessions, modified ADAS score was improved to 0 . This case particularly improved and almost all the skin lesions in treated arm have disappeared, whereas still mild erythema remained with pruritus yet on sham treated side. (e-h) Patient B, 28-years old man with modified ADAS score 18 on his CAP treated arm at week 0 and score 0 at week 4 . (i-l) Patient C, 20-years old woman with modified ADAS score 21 on her CAP treated shin at week 0 and score 15 at week 4.

\begin{tabular}{|l|l|l|l|l|}
\hline & W0 (baseline) & W1 & W2 & W4 \\
\hline Modified ADAS & $33.73 \pm 20.70$ & $21.56 \pm 15.70$ & $17.23 \pm 18.10$ & $13.12 \pm 15.60$ \\
\hline CAP & & & $<0.001^{*}$ \\
\hline$p$ value & & $28.45 \pm 15.00$ & $28.57 \pm 18.70$ & $27.39 \pm 23.20$ \\
\hline Sham & $35.02 \pm 14.80$ & & & $=0.114$ \\
\hline$p$-value & & $3.70 \pm 2.18$ & $3.05 \pm 2.16$ & $2.76 \pm 2.13$ \\
\hline EASI & $4.24 \pm 2.76$ & & & $=0.002$ \\
\hline$p$ value & & 6 & 7 & \\
\hline Number of patients showing improved IGA score & 15 \\
\hline CAP & N/A & 3 & 4 & 9 \\
\hline Sham & N/A & & 7 & \\
\hline
\end{tabular}

Table 2. Summary of clinical results. $A D A S$ atopic dermatitis antecubital severity, CAP cold atmospheric plasma, EASI eczema area and severity index, IGA investigator's global assessment.

been reported that the symptoms of pruritic skin improved significantly with CAP application for an average of 4.7 times a day for $2 \mathrm{~min}^{14}$. Moreover, CAP can promote the skin regeneration and wound healing by increasing the interleukin(IL)- 6 and transforming growth factor-beta (TGF-b) in the $\operatorname{skin}^{21,22}$. Moreover, stimulated angiogenesis during wound healing process was also noted by upregulating growth factors, such as vascular endothelial growth factor, epithelial growth factor, fibroblast growth factor, or by production of reactive oxygen 

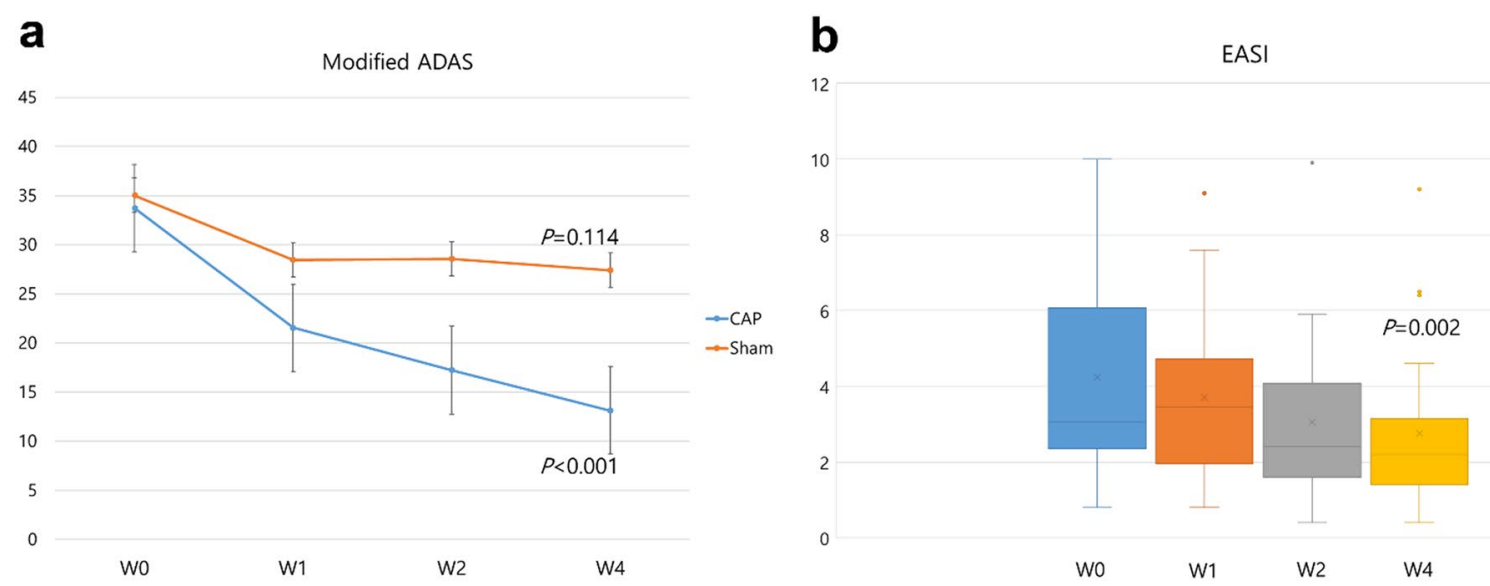

Figure 4. Clinical indexes along with follow-up period. (a) At week 4, modified ADAS score was significantly improved in plasma treated arm $(p$ value $<0.001)$, whereas score was not significantly changed in sham treated $\operatorname{arm}(p$ value $=0.114)$. (b) Patients' EASI score was significantly improved at week $4(p$ value $=0.002)$.

species $^{23}$. In the atopic mice skin, it was observed that the treatment of keratinocytes with CAP effectively downregulated the expression of CCL11, CCL13, and CCL17, which are main driving chemokines for the induction of $\mathrm{AD}^{24}$. In this human clinical study, patients with $\mathrm{AD}$ showed significant improvement in pruritic VAS ( $p$ value $=0.032)$ and atopic severity scores, including modified ADAS $(p<0.001)$, EASI $(p=0.002)$, and SCORAD score $(p$ value $=0.001)$. These results support that CAP would be helpful to relieve symptoms of AD by reducing chronic itching and promoting the recovery of secondary wounds caused by scratching.

Interestingly, CAP also revealed antibacterial effects in the skin. Isbary et al. reported that the bacteria in the wound area were reduced when an average of 7.86 treatments with CAP were performed, daily for 5 min for chronically unhealed wounds ${ }^{25}$. The bacteriocidal effect of CAP is meaningful in the treatment of AD, because the skin microbiome and its fluctuations are directly associated with $\mathrm{AD}^{26}$. Up to $90 \%$ of atopic patients showed dominant $S$. aureus colonization in skin microbiome and this increased colonization was known to be prominent in disease flare-up period ${ }^{27,28}$. Recent investigations in children suggested that cutaneous $S$. aureus colonization may lead to disease development, as well as, function as an exacerbating factor of $\mathrm{AD}^{29}$. Therefore, therapeutic attempts to restore the natural skin microflora by reducing colonized $S$. aureus are plausible strategies to improve the treatment of $\mathrm{AD}^{30,31}$. In this context, medications for $\mathrm{AD}$, such as topical corticosteroids and topical calcineurin inhibitors, play a role in restoring the diversity of skin microbiome ${ }^{32}$. In our microbiome analysis, the mean proportion of $S$. aureus was significantly decreased in CAP treated group comparative to sham treated group ( $p$ value $=0.047)$. Considering that atopic patients are susceptible to secondary bacterial infection due to a damaged skin barrier, the results of the present study are noteworthy. Indeed, CAP can improve atopic symptoms by promoting the recovery of the diversity of skin microbiome, as well as, to prevent secondary infection of the skin.

We attempted to demonstrate the therapeutic effect of CAP by measuring the change in TEWL as in the previous study ${ }^{33}$. However, the average decrease in TEWL was not significant before and after the treatment of CAP. The function of the skin barrier is affected by multiple extrinsic factors, such as temperature, humidity, and ultraviolet irradiation ${ }^{34,35}$. Recent studies have also revealed that the function of the skin barrier is associated with patients' stress ${ }^{36}$. Taken together these findings, there are some limitations in understanding the impact of CAP on the skin barrier function with changes of TEWL.

It is remarkable that there is a difference in response to CAP treatment according to total serum IgE. The group with total $\operatorname{IgE}>100 \mathrm{kU} / \mathrm{I}$ was significantly improved in modified ADAS score $(p$ value $<0.001)$ and EASI score $(p$ value $=0.002)$ after CAP treatments. These results are due to the difference between the extrinsic and intrinsic types, suggested subtypes of ADs. Extrinsic and intrinsic types are defined according to IgE-mediated sensitization, according to the presence of specific IgE for food and environmental allergens ${ }^{37}$. Since total serum IgE levels are significantly correlated with the allergen-specific IgE status ${ }^{38}$, total IgE is considered as a clinical marker to differentiate between the extrinsic and intrinsic types in both adults and children ${ }^{38,39}$. Thus, the group with total IgE $>100 \mathrm{kU} / \mathrm{I}$ are considered as extrinsic type of $\mathrm{AD}$ which has been described with elevated serum total IgE and disrupted skin barrier function ${ }^{37,40}$. Ricci et al. also found that the extrinsic type AD showed a higher colonization of $S$. aureus compared to the intrinsic type children $(71 \% \text { vs. } 49 \%)^{41}$. CAP treatment appears to improve the $\mathrm{AD}$ by restoring damaged skin barrier with antibacterial effect especially for causative $S$. aureus considering that CAP treatment is particularly effective in the group with elevated total IgE in this study, as above-mentioned.

The present pilot study has several limitations. First, this study was a single-center study with a small sample size. Further studies with multi-centered, large sample size are warranted. Second, patients with severe AD had not been included, because most of them were relied on oral immunosuppressants and reluctant to attempt CAP treatment alone. To evaluate the therapeutic effect of CAP alone, we considered that it is desirable to evaluate patients with mild to moderate $\mathrm{AD}$ in first place. Third, potential bias may have occurred due to the limited data on the microbiome analysis assessed in this study. Fourth, there is a possibility of "abscopal effect" and immunetriggering effect after the CAP treatment ${ }^{42}$. Nevertheless, our study is still noteworthy, because this is the first 
prospective clinical trial of CAP device on AD patients. Until now, there have been no clinical trials of CAP in human AD. In addition, CAP treatment is easy to apply in that it does not have safety problems at all, unlike oral immunosuppressant which have side effects in long term use. This strong point implies that CAP treatment also showed the potential as a burden-free adjuvant therapy. In fact, most of patients in this study showed a substantial satisfaction with CAP treatment without any discomfort. Combinatory treatment with topical agents would be effective treatment options, because CAP is also known to enhance cutaneous transdermal drug delivery by regulating E-cadherin-mediated cell junction ${ }^{43}$. Choi et al. demonstrated that combinatory treatment of CAP with conventional anti-inflammatory topical agents could be effective for shortening the healing duration and minimizing the drug amount ${ }^{24}$. In this study, the authors showed that the application of CAP or $1 \%$ hydrocortisone alone did not reduce the DNCB-mediated epidermal thickening in mice, but the thickened epidermis was restored by the combined treatment. Further clinical investigations with real human atopic skin are required to prove the effectiveness of CAP as an adjuvant treatment.

In conclusion, CAP has the potential to effectively improve the severity of mild and moderate AD by recovering the diversity of skin microbiome, as well as, promoting wound healing for damaged skin barrier. Patients' subjective pruritic symptoms will also be alleviated by the treatment of CAP without safety issues. This study supports new findings in treatment response data of CAP would update the new therapeutic approach in the AD field.

Received: 23 April 2021; Accepted: 28 June 2021

Published online: 14 July 2021

\section{References}

1. Heinlin, J. et al. Plasma medicine: Possible applications in dermatology. J. Dtsch. Dermatol. Ges. 8, 968-976 (2010).

2. Babaeva, N. Y. \& Kushner, M. J. Intracellular electric fields produced by dielectric barrier discharge treatment of skin. J. Phys. D Appl. Phys. 43, 185206. https://doi.org/10.1088/0022-3727/43/18/185206 (2010).

3. Gelker, M., Mrotzek, J., Ichter, A., Muller-Goymann, C. C. \& Viol, W. Influence of pulse characteristics and power density on stratum corneum permeabilization by dielectric barrier discharge. Biochim. Biophys. Acta Gen. Subj. 1863, 1513-1523 (2019).

4. Vijayarangan, V. et al. New insights on molecular internalization and drug delivery following plasma jet exposures. Int. J. Pharm. 589, 119874 (2020).

5. Isbary, G. et al. Successful and safe use of 2 min cold atmospheric argon plasma in chronic wounds: Results of a randomized controlled trial. Br. J. Dermatol. 167, 404-410 (2012).

6. Mirpour, S. et al. Cold atmospheric plasma as an effective method to treat diabetic foot ulcers: A randomized clinical trial. Sci. Rep. 10, 10440 (2020).

7. He, R. et al. The efficacy and safety of cold atmospheric plasma as a novel therapy for diabetic wound in vitro and in vivo. Int. Wound J. 17, 851-863 (2020).

8. Kisch, T. et al. The repetitive use of non-thermal dielectric barrier discharge plasma boosts cutaneous microcirculatory effects. Microvasc. Res. 106, 8-13 (2016).

9. Collet, G. et al. Plasma jet-induced tissue oxygenation: Potentialities for new therapeutic strategies. Plasma Sources Sci. Technol. 23, 012005 (2014).

10. Lee, M. H. et al. Non-thermal plasma inhibits mast cell activation and ameliorates allergic skin inflammatory diseases in NC/Nga mice. Sci. Rep. 9, 13510 (2019).

11. Busco, G., Robert, E., Chettouh-Hammas, N., Pouvesle, J. M. \& Grillon, C. The emerging potential of cold atmospheric plasma in skin biology. Free Radic. Biol. Med. 161, 290-304 (2020).

12. Larsen, F. S. \& Hanifin, J. M. Epidemiology of atopic dermatitis. Immunol. Allergy Clin. 22, 1-24 (2002).

13. Gareri, C., Bennardo, L. \& De Masi, G. Use of a new cold plasma tool for psoriasis treatment: A case report. SAGE Open Med. Case Rep. 8, 2050313X20922709 (2020).

14. Heinlin, J. et al. A randomized two-sided placebo-controlled study on the efficacy and safety of atmospheric non-thermal argon plasma for pruritus. J. Eur. Acad. Dermatol. Venereol. JEADV 27, 324-331 (2013).

15. Hwang, S. G., Kim, J. H., Jo, S. Y., Kim, Y. J. \& Won, C. H. Cold atmospheric plasma prevents wrinkle formation via an antiaging process. Plasma Med. 10, 91-102 (2020).

16. Eom, I. S., Kang, H. Y. \& Kwon, S. K. Plasma diagnosis and biomedical application using linear microwave atmospheric-pressure plasma generator. IEEE Trans. Plasma Sci. 48, 3054-3060 (2020).

17. Baek, J. H. et al. The atopic dermatitis antecubital severity score: Validity, reliability, and sensitivity to change in patients with atopic dermatitis. Int. J. Dermatol. 54, 1382-1389 (2015).

18. Reich, A. et al. Visual analogue scale: Evaluation of the instrument for the assessment of pruritus. Acta Derm. Venereol. 92, 497-501 (2012).

19. Kapur, S., Watson, W. \& Carr, S. Atopic dermatitis. Allergy Asthma Clin. Immunol. 14, 52 (2018).

20. Buddenkotte, J. \& Steinhoff, M. Pathophysiology and therapy of pruritus in allergic and atopic diseases. Allergy 65, 805-821 (2010).

21. Arndt, S. et al. Cold atmospheric plasma (CAP) changes gene expression of key molecules of the wound healing machinery and improves wound healing in vitro and in vivo. PLOS ONE 8, e79325 (2013).

22. Fathollah, S. et al. Investigation on the effects of the atmospheric pressure plasma on wound healing in diabetic rats. Sci. Rep. 6, 19144 (2016).

23. Arndt, S., Unger, P., Berneburg, M., Bosserhoff, A. K. \& Karrer, S. Cold atmospheric plasma (CAP) activates angiogenesis-related molecules in skin keratinocytes, fibroblasts and endothelial cells and improves wound angiogenesis in an autocrine and paracrine mode. J. Dermatol. Sci. 89, 181-190 (2018).

24. Choi, J. H., Song, Y. S., Lee, H. J., Hong, J. W. \& Kim, G. C. Inhibition of inflammatory reactions in 2,4-Dinitrochlorobenzene induced Nc/Nga atopic dermatitis mice by non-thermal plasma. Sci. Rep. 6, 27376 (2016).

25. Isbary, G. et al. A first prospective randomized controlled trial to decrease bacterial load using cold atmospheric argon plasma on chronic wounds in patients. Br. J. Dermatol. 163, 78-82 (2010).

26. Nakamura, Y. et al. Staphylococcus delta-toxin induces allergic skin disease by activating mast cells. Nature 503, 397-401 (2013).

27. Leyden, J. J., Marples, R. R. \& Kligman, A. M. Staphylococcus aureus in the lesions of atopic dermatitis. Br. J. Dermatol. 90, 525-530 (1974).

28. Park, H. Y. et al. Staphylococcus aureus colonization in acute and chronic skin lesions of patients with atopic dermatitis. Ann. Dermatol. 25, 410-416 (2013). 
29. Meylan, P. et al. Skin colonization by Staphylococcus aureus precedes the clinical diagnosis of atopic dermatitis in Infancy. J. Invest. Dermatol. 137, 2497-2504 (2017).

30. Salava, A. \& Lauerma, A. Role of the skin microbiome in atopic dermatitis. Clin. Transl. Allergy 4, 33 (2014).

31. Wong, S. M., Ng, T. G. \& Baba, R. Efficacy and safety of sodium hypochlorite (bleach) baths in patients with moderate to severe atopic dermatitis in Malaysia. J. Dermatol. 40, 874-880 (2013).

32. Kim, J. E. \& Kim, H. S. Microbiome of the skin and gut in atopic dermatitis (AD): Understanding the pathophysiology and finding novel management strategies. J. Clin. Med. 8, 444 (2019).

33. Schmidt, A. et al. The molecular and physiological consequences of cold plasma treatment in murine skin and its barrier function. Free Radic. Biol. Med. 161, 32-49 (2020).

34. Engebretsen, K. A., Johansen, J. D., Kezic, S., Linneberg, A. \& Thyssen, J. P. The effect of environmental humidity and temperature on skin barrier function and dermatitis. J. Eur. Acad. Dermatol. Venereol. JEADV 30, 223-249 (2016).

35. Jiang, S. J. et al. Biophysical and morphological changes in the stratum corneum lipids induced by UVB irradiation. J. Dermatol. Sci. 44, 29-36 (2006).

36. Maarouf, M., Maarouf, C. L., Yosipovitch, G. \& Shi, V. Y. The impact of stress on epidermal barrier function: An evidence-based review. Br. J. Dermatol. 181, 1129-1137 (2019).

37. Tokura, Y. Extrinsic and intrinsic types of atopic dermatitis. J. Dermatol. Sci. 58, 1-7 (2010).

38. Ott, H. et al. Total serum IgE as a parameter to differentiate between intrinsic and extrinsic atopic dermatitis in children. Acta Derm. Venereol. 89, 257-261 (2009).

39. Mori, T. et al. Comparison of skin barrier function and sensory nerve electric current perception threshold between IgE-high extrinsic and IgE-normal intrinsic types of atopic dermatitis. Br. J. Dermatol. 162, 83-90 (2010).

40. Kabashima, K. New concept of the pathogenesis of atopic dermatitis: Interplay among the barrier, allergy, and pruritus as a trinity. J. Dermatol. Sci. 70, 3-11 (2013).

41. Ricci, G., Patrizi, A., Neri, I., Bendandi, B. \& Masi, M. Frequency and clinical role of Staphylococcus aureus overinfection in atopic dermatitis in children. Pediatr. Dermatol. 20, 389-392 (2003).

42. Mahdikia, H. et al. Gas plasma irradiation of breast cancers promotes immunogenicity, tumor reduction, and an abscopal effect in vivo. Oncoimmunology 10, 1859731 (2020).

43. Choi, J. H. et al. Treatment with low-temperature atmospheric pressure plasma enhances cutaneous delivery of epidermal growth factor by regulating E-cadherin-mediated cell junctions. Arch. Dermatol. Res. 306, 635-643 (2014).

\section{Acknowledgements}

This study was approved by the institutional review board of Asan Medical Center (2020-0811) and followed the guidelines of the 1975 Declaration of Helsinki. An informed consent was obtained from all patients before their participation in the study.

\section{Author contributions}

Y.J.K., D.J.L., M.Y.L., W.J.L. S.E.C. and C.H.W. had full access to all the data in the study and take responsibility for the integrity of the data and the accuracy of the data analysis. Concept and design, data acquisition, data analysis/interpretation, drafting manuscript, critical revision of manuscript, and final approval: Y.J.K., D.J.L., M.Y.L., W.J.L., S.E.C. and C.H.W. Statistical analysis: Y.J.K. and D.J.L. Technical support: W.J.L. and S.E.C. Supervision: C.H.W. All authors reviewed the manuscript.

\section{Competing interests}

The authors declare no competing interests.

\section{Additional information}

Supplementary Information The online version contains supplementary material available at https:/doi.org/ 10.1038/s41598-021-93941-y.

Correspondence and requests for materials should be addressed to C.H.W.

Reprints and permissions information is available at www.nature.com/reprints.

Publisher's note Springer Nature remains neutral with regard to jurisdictional claims in published maps and institutional affiliations.

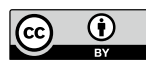

Open Access This article is licensed under a Creative Commons Attribution 4.0 International License, which permits use, sharing, adaptation, distribution and reproduction in any medium or format, as long as you give appropriate credit to the original author(s) and the source, provide a link to the Creative Commons licence, and indicate if changes were made. The images or other third party material in this article are included in the article's Creative Commons licence, unless indicated otherwise in a credit line to the material. If material is not included in the article's Creative Commons licence and your intended use is not permitted by statutory regulation or exceeds the permitted use, you will need to obtain permission directly from the copyright holder. To view a copy of this licence, visit http://creativecommons.org/licenses/by/4.0/.

(C) The Author(s) 2021 